\title{
NORM EQUIVALENCE AND COMPOSITION OPERATORS BETWEEN BLOCH/LIPSCHITZ SPACES OF THE BALL
}

\author{
DANA D. CLAHANE AND STEVO STEVIĆ
}

Received 11 October 2005; Revised 30 January 2006; Accepted 12 February 2006

For $p>0$, let $\mathscr{B}^{p}\left(\mathbb{B}_{n}\right)$ and $\mathscr{L}_{p}\left(\mathbb{B}_{n}\right)$ denote, respectively, the $p$-Bloch and holomorphic $p$-Lipschitz spaces of the open unit ball $\mathbb{B}_{n}$ in $\mathbb{C}^{n}$. It is known that $\mathscr{B}^{p}\left(\mathbb{B}_{n}\right)$ and $\mathscr{L}_{1-p}\left(\mathbb{B}_{n}\right)$ are equal as sets when $p \in(0,1)$. We prove that these spaces are additionally normequivalent, thus extending known results for $n=1$ and the polydisk. As an application, we generalize work by Madigan on the disk by investigating boundedness of the composition operator $\mathfrak{C}_{\phi}$ from $\mathscr{L}_{p}\left(\mathbb{B}_{n}\right)$ to $\mathscr{L}_{q}\left(\mathbb{B}_{n}\right)$.

Copyright (c) 2006 D. D. Clahane and S. Stević. This is an open access article distributed under the Creative Commons Attribution License, which permits unrestricted use, distribution, and reproduction in any medium, provided the original work is properly cited.

\section{Background and terminology}

Let $n \in \mathbb{N}$, and suppose that $\mathbb{D}$ is a domain in $\mathbb{C}^{n}$. Denote the linear space of complexvalued, holomorphic functions on $\mathbb{D}$ by $\mathscr{H}(\mathbb{D})$. If $\mathscr{X}$ is a linear subspace of $\mathscr{H}(\mathbb{D})$ and $\phi: \mathbb{D} \rightarrow \mathbb{D}$ is holomorphic, then one can define the linear operator $\mathfrak{C}_{\phi}: \mathscr{X} \rightarrow \mathscr{H}(\mathbb{D})$ by $\mathfrak{C}_{\phi}(f)=f \circ \phi$ for all $f \in \mathscr{X} \cdot \mathfrak{C}_{\phi}$ is called the composition operator induced by $\phi$.

The problem of relating properties of symbols $\phi$ and operators such as $\mathfrak{C}_{\phi}$ that are induced by these symbols is of fundamental importance in concrete operator theory. However, efforts to obtain characterizations of self-maps that induce bounded composition operators on many function spaces have not yielded completely satisfactory results in the several-variable case, leaving a wealth of basic open problems.

In this paper, we try to make progress toward the goal of characterizing the holomorphic self-maps of the open unit ball $\mathbb{B}_{n}$ in $\mathbb{C}^{n}$ that induce bounded composition operators between holomorphic $p$-Lipschitz spaces $\mathscr{L}_{p}\left(\mathbb{B}_{n}\right)$ for $0<p<1$ by translating the problem to $(1-p)$-Bloch spaces $\mathscr{B}^{1-p}\left(\mathbb{B}_{n}\right)$ via an auxiliary Hardy/Littlewood-type norm-equivalence result of potential independent interest. This method was also used in [7] for $\mathbb{B}_{1}$ and in [3] for the unit polydisk $\Delta^{n}$.

The function-theoretic characterization of analytic self-maps of $\mathbb{B}_{1}$ that induce bounded composition operators on $\mathscr{L}_{p}\left(\mathbb{B}_{1}\right)$ for $0<p<1$ is due to Madigan [7], and the case of 
$\Delta^{n}$ was handled in a joint paper by the present authors with Zhou [3], in which a full characterization of the holomorphic self-maps $\phi$ of $\Delta^{n}$ that induce bounded composition operators between $\mathscr{L}_{p}\left(\Delta^{n}\right)$ and $\mathscr{L}_{q}\left(\Delta^{n}\right)$, and, more generally, between Bloch spaces $\mathscr{B}^{p}\left(\Delta^{n}\right)$ and $\mathscr{B} q\left(\Delta^{n}\right)$, is obtained for $p, q \in(0,1)$, along with analogous characterizations of compact composition operators between these spaces.

Although our main results concerning composition operators, Theorem 2.1 and Corollary 2.2, are not full characterizations, they do generalize Madigan's result for the disk to $\mathbb{B}_{n}$; on the other hand, we obtain a complete Hardy-Littlewood norm-equivalence result for $p$-Bloch and $(1-p)$-Lipschitz spaces of $\mathbb{B}_{n}$ for all $n \in \mathbb{N}$. This norm-equivalence result should lead to an eventual extension to $\mathbb{B}_{n}$ of the characterizations of bounded composition operators established on $\mathbb{B}_{1}$ in [7] and on $\Delta^{n}$ in [3].

Most of our several complex variables notation is adopted from [8]. If $z=\left(z_{1}, \ldots, z_{n}\right)$ and $w=\left(w_{1}, \ldots, w_{n}\right)$ are points in $\mathbb{C}^{n}$, then we define a complex inner product by $\langle z, \omega\rangle=$ $\sum_{k=1}^{n} z_{k} \bar{w}_{k}$ and put $|z|:=\sqrt{\langle z, z\rangle}$. We call $\mathbb{B}_{n}:=\left\{z \in \mathbb{C}^{n}:|z|<1\right\}$ the (open) unit ball of $\mathbb{C}^{n}$.

Let $p \in(0, \infty)$. The $p$-Bloch space $\mathscr{B}_{P} p\left(\mathbb{B}_{n}\right)$ consists of all $f \in \mathscr{H}\left(\mathbb{B}_{n}\right)$ with the property that there is an $M \geq 0$ such that

$$
b(f, z, p):=\left(1-|z|^{2}\right)^{p}|\nabla f(z)| \leq M \quad \forall z \in \mathbb{B}_{n} .
$$

$\mathscr{B}_{P} P\left(\mathbb{B}_{n}\right)$ is a Banach space with norm $\|f\|_{\mathscr{R}^{p}}$ given by

$$
\|f\|_{\Re^{p}}=|f(0)|+\sup _{z \in \mathbb{B}_{n}} b(f, z, p) .
$$

The little $p$-Bloch space $\mathscr{B}_{0}^{p}\left(\mathbb{B}_{n}\right)$ is defined as the closed subspace of $\mathscr{B}^{p}\left(\mathbb{B}_{n}\right)$ consisting of the functions that satisfy

$$
\lim _{z \rightarrow \partial \mathbb{B}_{n}}\left(1-|z|^{2}\right)^{p}|\nabla f(z)|=0
$$

For $p \in(0,1), \mathscr{L}_{p}\left(\mathbb{B}_{n}\right)$ denotes the holomorphic p-Lipschitz space which is the set of all $f \in \mathscr{H}\left(\mathbb{B}_{n}\right)$ such that for some $C>0$,

$$
|f(z)-f(w)| \leq C|z-w|^{p} \quad \text { for every } z, w \in \mathbb{B}_{n} .
$$

These functions extend continuously to $\overline{\mathbb{B}}_{n}$ (cf. [3, Lemma 4.4]). Therefore, if $A\left(\mathbb{B}_{n}\right)$ is the ball algebra $[8$, Chapter 6$]$, then

$$
\mathscr{L}_{p}\left(\mathbb{B}_{n}\right)=\operatorname{Lip}_{p}\left(\mathbb{B}_{n}\right) \cap A\left(\mathbb{B}_{n}\right)
$$

where $\operatorname{Lip}_{p}\left(\mathbb{B}_{n}\right)$ is the set of all $f: \mathbb{B}_{n} \rightarrow \mathbb{C}$ satisfying (1.4) for some $C>0$ and all $z \in \overline{\mathbb{B}}_{n}$. $\mathscr{L}_{p}\left(\mathbb{B}_{n}\right)$ is endowed with a complete norm $\|\cdot\|_{\mathscr{L}_{p}}$ that is given by

$$
\|f\|_{\mathscr{L}_{p}}=|f(0)|+\sup _{z \neq w: z, w \in \mathbb{B}_{n}}\left\{\frac{|f(z)-f(w)|}{|z-w|^{p}}\right\} .
$$


In (1.4) and (1.6), $\mathbb{B}_{n}$ and $\overline{\mathbb{B}}_{n}$ are interchangeable, since functions in $\mathscr{L}_{p}\left(\mathbb{B}_{n}\right)$ extend continuously to $\overline{\mathbb{B}}_{n}$. The supremum above is called the Lipschitz constant for $f$. As in [8, page 13], $\sigma$ represents the unique rotation-invariant positive Borel measure on $\partial \mathbb{B}_{n}$ for which $\sigma\left(\partial \mathbb{B}_{n}\right)=1$, and for $f \in L^{1}(\sigma), C[f]$ denotes Cauchy integral of $f$ on $\mathbb{B}_{n}$ (see $[8$, page 38]).

Let $u \in \partial \mathbb{B}_{n}$ and $f \in \mathscr{H}\left(\mathbb{B}_{n}\right)$. The directional derivative of $f$ at $z \in \mathbb{B}_{n}$ in the direction of $u \in \partial \mathbb{B}_{n}$ is given by

$$
D_{u} f(z)=\lim _{\lambda \rightarrow 0, \lambda \in \mathbb{C}} \frac{f(z+\lambda u)-f(z)}{\lambda} .
$$

Observe that

$$
D_{u} f(z)=\langle\nabla f(z), \bar{u}\rangle
$$

We define the partial differential operators $D_{j}$ as in $[8$, Chapter 1]. The radial derivative operator $[8$, page 103$]$ in $\mathbb{C}^{n}$ will be denoted by $\mathfrak{R}$ and is linear. Let $\mathbb{U}=\left\{u_{1}, u_{2}, \ldots, u_{n}\right\}$ be an orthonormal basis for the Hilbert space $\mathbb{C}^{n}$ with its usual Euclidean structure. We define a gradient operator $\nabla^{\mathbb{U}}$ on $\mathscr{H}(\mathbb{D})$ with respect to $\mathbb{U}$ by

$$
\nabla^{\cup} f(z)=\left(D_{u_{1}} f(z), D_{u_{2}} f(z), \ldots, D_{u_{n}} f(z)\right),
$$

and we denote $\nabla^{\mathbb{U}}$ by $\nabla$ when $\mathbb{U}$ is the typically ordered standard basis for $\mathbb{C}^{n}$.

Let $x$ and $y$ be two positive variable quantities. We write $x \asymp y$ (and say that $x$ and $y$ are comparable) if and only if $x / y$ is bounded above and below.

\section{Main results on composition operators}

Our norm-equivalence result (Theorem 3.5) ties our results concerning $\mathfrak{C}_{\phi}$ between $p$-Lipschitz spaces of $\mathbb{B}_{n}$ to the following result for general Bloch spaces.

Theorem 2.1. Let $p, q \in(0, \infty)$, and suppose that $\phi: \mathbb{B}_{n} \rightarrow \mathbb{B}_{n}$ is holomorphic. Then the following statements hold.

(A) If there is an $M \geq 0$ such that for all $z \in \mathbb{B}_{n}$ and $j \in\{1, \ldots, n\}$,

$$
\frac{\left(1-|z|^{2}\right)^{q}}{\left(1-|\phi(z)|^{2}\right)^{p}}\left|\nabla \phi_{j}(z)\right| \leq M
$$

then $\mathfrak{C}_{\phi}$ is bounded from $\mathscr{B}^{p}\left(\mathbb{B}_{n}\right)$ (resp., $\mathscr{B}_{0}^{p}\left(\mathbb{B}_{n}\right)$ ) to $\mathscr{B}^{q}\left(\mathbb{B}_{n}\right)$.

(B) If $\mathfrak{C}_{\phi}$ is bounded from $\mathscr{B}^{p}\left(\mathbb{B}_{n}\right)$ (resp., $\mathscr{B}_{0}^{p}\left(\mathbb{B}_{n}\right)$ ) to $\mathscr{B}^{q}\left(\mathbb{B}_{n}\right)$, then there is an $M^{\prime} \geq 0$ such that for all $z \in \mathbb{B}_{n}$ and $u \in \partial \mathbb{B}_{n}$,

$$
\frac{\left(1-|z|^{2}\right)^{q}}{\left(1-|\langle\phi(z), u\rangle|^{2}\right)^{p}}|\nabla\langle\phi(z), u\rangle| \leq M^{\prime}
$$


Theorem 2.1 above and Corollary 2.2 below for $0<p=q<1$ appear in [2, Chapter 4]. It should be pointed out that Theorem 2.1, part (A) is similar to a statement that is proved in [11]; furthermore, [11] contains a result that is in the same direction as part (B) of Theorem 2.1 and that is proven using different testing functions. Unlike [11], however, the present paper addresses composition operators between $\mathscr{L}_{p}\left(\mathbb{B}_{n}\right)$ and $\mathscr{L}_{q}\left(\mathbb{B}_{n}\right)$ and the coincidence and norm equivalence of $\mathscr{S}^{1-p}\left(\mathbb{B}_{n}\right)$ and $\mathscr{L}_{p}\left(\mathbb{B}_{n}\right)$, respectively.

It is natural to consider the application of corresponding "little-oh" arguments to obtain a compactness result analogous to Theorem 2.1, in which "bounded" is replaced by "compact" and the limit of the left-hand side of each inequality in the statement is taken as $|\phi(z)| \rightarrow 1^{-}$, with inequality replaced by equality to 0 . However, in the case, that $p \in(0,1), \mathscr{B}^{p}\left(\mathbb{B}_{n}\right)$ is the same as and norm-equivalent to $\mathscr{L}_{1-p}\left(\mathbb{B}_{n}\right)$, whose compact composition operators are known (by a result due to J. H. Shapiro) to be generated precisely by holomorphic self-maps $\phi$ of $\mathbb{B}_{n}$ with supremum norm strictly less than 1 (see [4, Chapter 4]).

The following corollary follows from Theorems 2.1 and 3.5 and extends the main result of [7].

Corollary 2.2. Let $p, q \in(0,1)$, and suppose that $\phi: \mathbb{B}_{n} \rightarrow \mathbb{B}_{n}$ is holomorphic. Then the following statements hold.

(A) If there is an $M \geq 0$ such that

$$
\frac{\left(1-|z|^{2}\right)^{1-q}}{\left(1-|\phi(z)|^{2}\right)^{1-p}}\left|\nabla \phi_{j}(z)\right| \leq M,
$$

for all $j \in\{1,2 \ldots, n\}$ and $z \in \mathbb{B}_{n}$, then $\mathfrak{C}_{\phi}$ is a bounded operator from $\mathscr{L}_{p}\left(\mathbb{B}_{n}\right)$ to $\mathscr{L}_{q}\left(\mathbb{B}_{n}\right)$.

(B) If $\mathfrak{C}_{\phi}$ is a bounded operator from $\mathscr{L}_{p}\left(\mathbb{B}_{n}\right)$ to $\mathscr{L}_{q}\left(\mathbb{B}_{n}\right)$, then there is an $M^{\prime} \geq 0$ such that for all $z \in \mathbb{B}_{n}$ and $u \in \partial \mathbb{B}_{n}$,

$$
\frac{\left(1-|z|^{2}\right)^{1-q}}{\left(1-|\langle\phi(z), u\rangle|^{2}\right)^{1-p}}|\nabla\langle\phi(z), u\rangle| \leq M^{\prime} .
$$

Choosing $n=1, p=q \in(0,1)$, and $u=1$ in Corollary 2.2 leads to the following result, which appears in [7].

Theorem 2.3. Let $0<p<1$, and suppose that $\phi$ is an analytic self-map of $\mathbb{B}_{1}$. Then $\mathfrak{C}_{\phi}$ is bounded on $\mathscr{L}_{p}\left(\mathbb{B}_{1}\right)$ if and only if

$$
\sup _{z \in \mathbb{B}_{1}}\left\{\left(\frac{1-|z|^{2}}{1-|\phi(z)|}\right)^{1-p}\left|\phi^{\prime}(z)\right|\right\}<\infty .
$$

3. Norm equivalence of $\mathscr{L}_{p}\left(\mathbb{B}_{n}\right)$ and $\mathscr{B}^{1-p}\left(\mathbb{B}_{n}\right)$ for $0<p<1$

To generalize Theorem 2.3 to $\mathbb{B}_{n}$, we need Theorem 3.5, which is the ball analogue of the following result for the disk [7, Lemma 2]. The first statement in Theorem 3.1 can be 
derived from a classical theorem of Hardy/Littlewood for $n=1$ (see [6], [5, page 74], and [4, page 176]).

Theorem 3.1. Let $0<p<1$. If $f: \mathbb{B}_{1} \rightarrow \mathbb{C}$ is analytic, then $f \in \mathscr{L}_{p}\left(\mathbb{B}_{1}\right)$ if and only if

$$
\left|f^{\prime}(z)\right|=O\left(\frac{1}{1-|z|^{2}}\right)^{1-p}
$$

Furthermore, the Lipschitz constant of $f$ and the quantity

$$
\sup _{z \in \mathbb{B}_{1}}\left\{\left(1-|z|^{2}\right)^{1-p}\left|f^{\prime}(z)\right|\right\}
$$

are comparable as $f$ varies through $\mathscr{L}_{p}\left(\mathbb{B}_{1}\right)$.

We remark that the polydisk version of Theorem 3.1 is stated and proved in [3]. However, the argument used there cannot be applied to $\mathbb{B}_{n}$, so we need a different approach for that domain. We will proceed by listing some lemmas, which together essentially form the norm-equivalence result, Theorem 3.5.

For $0<p<1$, we define $\|\cdot\|_{\Re^{1-p}}^{\Re}$ on $\mathscr{L}_{p}\left(\mathbb{B}_{n}\right)$ by

$$
\|f\|_{\mathscr{R}^{1-p}}^{\Re}=|f(0)|+\sup _{z \in \mathbb{B}_{n}}\left\{\left(1-|z|^{2}\right)^{1-p}|(\Re f)(z)|\right\} .
$$

It can be shown by subsequent applications of Lemmas 3.2 and 3.3 below that $\|\cdot\|_{\mathscr{R}^{1-p}}^{\mathscr{R}}$ is a norm on $\mathscr{L}_{p}\left(\mathbb{B}_{n}\right)$.

We start with the following lemma.

Lemma 3.2. Suppose that $0<p<1$. Then $\mathscr{L}_{p}\left(\mathbb{B}_{n}\right) \subset \mathscr{B}^{1-p}$. Furthermore, there is a $C_{p} \geq 0$ such that for all $f \in \mathscr{L}_{p}\left(\mathbb{B}_{n}\right)$,

$$
\|f\|_{\mathscr{R}^{1-p}}^{\mathfrak{R}} \leq C_{p}\|f\|_{\mathscr{L}_{p}}
$$

Proof. The proof of the first statement is standard and left to the reader. Since functions in $\mathscr{L}_{p}\left(\mathbb{B}_{n}\right)$ extend continuously to $\overline{\mathbb{B}}_{n}$, they are automatically in $L^{1}(\sigma)$ [8, Remark, page 107] and since the quotients of these functions and their $\mathscr{L}_{p}$-norms satisfy [8, equation (1), page 107], the second statement is obtained from [8, Theorem 6.4.9].

The following lemma is also a portion of Theorem 3.5.

Lemma 3.3. If $p \in(0,1)$, then $\mathscr{B}^{1-p}\left(\mathbb{B}_{n}\right) \subset \mathscr{L}_{p}\left(\mathbb{B}_{n}\right)$, and

$$
\|f\|_{\mathscr{L}_{p}} \leq\left(2+2 p^{-1}\right)\|f\|_{\mathscr{B}^{1-p}} \quad \forall f \in \mathscr{B}^{1-p}\left(\mathbb{B}_{n}\right)
$$

Proof. Suppose that $f \in \mathscr{B}^{1-p}\left(\mathbb{B}_{n}\right)$. If $f=0$, then $f \in \mathscr{L}_{p}\left(\mathbb{B}_{n}\right)$ trivially, so assume henceforward that $f \neq 0$. A well-known result [8, Chapter 6] applied to $f /\|f\|_{\Re^{1-p}}$ implies that 
6 Norm equivalence and composition operators

for all $z, w \in B_{n}$,

$$
\frac{1}{\|f\|_{\Re^{1-p}}}|f(z)-f(w)| \leq\left(1+2 p^{-1}\right)|z-w|^{p}
$$

from which the first statement of the lemma follows. Moreover,

$$
\begin{aligned}
\|f\|_{\mathscr{L}_{p}} & =|f(0)|+\sup _{z, w \in \mathbb{B}_{n}: z \neq w} \frac{|f(z)-f(w)|}{|z-w|^{p}} \\
& \leq|f(0)|+\left(1+2 p^{-1}\right)\|f\|_{\mathscr{B}^{1-p}} \leq\left(2+2 p^{-1}\right)\|f\|_{\mathscr{B}^{1-p}} .
\end{aligned}
$$

The following fact also constitutes a part of Theorem 3.5.

Lemma 3.4. Let $p>0$. Then $f \in \mathscr{B}^{P}\left(\mathbb{B}_{n}\right)$ if and only if there is an $M \geq 0$ such that for all $z \in \mathbb{B}_{n},|(\Re f)(z)|\left(1-|z|^{2}\right)^{p} \leq M$. If $p \in(0,1]$, then there is a $C_{p} \geq 0$ such that $\|f\|_{\Re^{p}} \leq$ $C_{p}\|f\|_{\Re^{p}}^{\Re}$ for all $f \in \mathscr{B}^{p}\left(\mathbb{B}_{n}\right)$.

Proof. For a proof of the first statement, see [10, Proposition 1]. To prove the second statement, we use the weighted Bergman projection $P_{s}$ with kernel $K_{s}$ and the map $L_{s}$ defined on $P_{s}\left[L^{\infty}\left(B_{n}\right)\right]$ by

$$
\left(L_{s} g\right)(z)=(s+1)^{-1}\left(1-|z|^{2}\right)[(n+s+1) g(z)+(\Re g)(z)] \quad \forall z \in \mathbb{B}_{n}
$$

where $s \in \mathbb{C}$ satisfies Res $>-1$ (see [1]). By [1, Corollary 13], we have that $P_{s} \circ L_{s}$ is the identity on $\mathscr{B}^{1}\left(\mathbb{B}_{n}\right)$ for all such values of $s$. In particular, $P_{0} \circ L_{0}$ is the identity on $\mathscr{S}^{P}\left(\mathbb{B}_{n}\right)$, since this set is contained in $\mathscr{B}^{1}\left(\mathbb{B}_{n}\right)$. Note that the assumption $p \in(0,1]$ is used here.

We then obtain that there is a $C \geq 0$ such that for all $z \in \mathbb{B}_{n}$ and $f \in \mathscr{B}^{1}\left(\mathbb{B}_{n}\right)$,

$$
f(z)=\left(P_{0} \circ L_{0}\right)(f)(z)=C \int_{\mathbb{B}_{n}}\left(1-|w|^{2}\right) K_{0}(z, w)[(n+1) f(w)+\Re f(w)] d V(w) .
$$

Hence, there is a $C^{\prime} \geq 0$ such that for all $f \in \mathscr{B}^{P}\left(\mathbb{B}_{n}\right)$ and $z \in \mathbb{B}_{n}$,

$$
\begin{aligned}
|\nabla f(z)| \leq & C^{\prime} \int_{\mathbb{B}_{n}}\left(1-|w|^{2}\right)\left|\nabla K_{0}(z, w)\right||f(w)| d V(w) \\
& +C^{\prime} \int_{\mathbb{B}_{n}}\left(1-|w|^{2}\right)\left|\nabla K_{0}(z, w)\right||\Re f(w)| d V(w) .
\end{aligned}
$$

Let $\varepsilon \in(1-p, 1)$. Subsequent applications of the above inequality (see [9, Lemma 2] and $\left[8\right.$, Theorem 1.4.10]) imply that there are nonnegative constants $C^{\prime \prime}$ and $C^{\prime \prime \prime}$ such that 
for all $z \in B_{n}$ and $f \in \mathscr{B}^{P}\left(\mathbb{B}_{n}\right)$, the following chain of inequalities holds:

$$
\begin{aligned}
|\nabla f(z)| \leq & C^{\prime} \int_{\mathbb{B}_{n}} \frac{\left(1-|w|^{2}\right)|w|}{|1-\langle z, w\rangle|^{n+2}}|f(w)| d V(w) \\
& +C^{\prime} \int_{\mathbb{B}_{n}} \frac{\left(1-|w|^{2}\right)|w|}{|1-\langle z, w\rangle|^{n+2}}|\Re f(w)| d V(w) \\
\leq & C^{\prime \prime}\|f\|_{\mathscr{R}^{p}}^{\Re} \int_{\mathbb{B}_{n}} \frac{\left(1-|w|^{2}\right)^{\varepsilon}}{|1-\langle z, w\rangle|^{n+2}} d V(w) \\
& +C^{\prime \prime}\|f\|_{\mathscr{R}^{p}}^{\Re} \int_{\mathbb{B}_{n}} \frac{\left(1-|w|^{2}\right)^{1-p}}{|1-\langle z, w\rangle|^{n+2}} d V(w) \\
\leq & C^{\prime \prime \prime}\|f\|_{\mathscr{R}^{p}}^{\Re} \frac{1}{(1-|z|)^{1-\varepsilon}}+C^{\prime \prime \prime}\|f\|_{\mathscr{R}^{p}}^{\Re} \frac{1}{(1-|z|)^{p}} .
\end{aligned}
$$

It follows that for all $f \in \mathscr{S}_{P} P\left(\mathbb{B}_{n}\right)$ and $z \in \mathbb{B}_{n}$,

$$
\left(1-|z|^{2}\right)^{p}|\nabla f(z)| \leq 2^{p+1} C^{\prime \prime \prime}\|f\|_{\Re^{p}}^{\Re}
$$

The second statement in the lemma now follows from the above statement and an application of [9, Lemma 2] at $z=0$.

Next, we state and prove this section's main result, the analogue of Theorem 3.1 for $\mathbb{B}_{n}$. We emphasize that while the statement of equality in the theorem is known and can be obtained, for example, from [12], the norm-equivalence portion requires additional work that includes the previous lemmas and the proof below. Furthermore, neither this result nor its proof has appeared previously in any literature that is known to the authors, though it seems to be part of the folklore. The proof of this rather fundamental theorem seems to be nontrivial and worthy of recording.

Theorem 3.5. If $0<p<1$, then $\mathscr{B}^{1-p}\left(\mathbb{B}_{n}\right)=\mathscr{L}_{p}\left(\mathbb{B}_{n}\right)$; furthermore,

$$
\|f\|_{\Re^{1-p}} \asymp\|f\|_{\Re^{1-p}}^{\Re} \asymp\|f\|_{\mathscr{L}_{p}}
$$

as $f$ varies through $\mathscr{L}_{p}\left(\mathbb{B}_{n}\right)$.

Proof. The first statement is known, since $\mathscr{L}_{p}\left(\mathbb{B}_{n}\right)=A\left(\mathbb{B}_{n}\right) \cap \operatorname{Lip}_{\alpha}\left(\mathbb{B}_{n}\right)$ (see $[8$, Chapter $6]$ ), which is set theoretically equal to $\mathscr{B}^{1-}{ }^{1-}\left(\mathbb{B}_{n}\right)$ (see [10]). By Lemma 3.4, it follows that there is a $C_{p} \geq 0$ such that for all $f \in \mathscr{L}_{p}\left(\mathbb{B}_{n}\right),\|f\|_{\mathscr{R}^{1-p}} \leq C_{p}\|f\|_{\Re^{1-p}}^{\Re}$. It follows from Lemma 3.2 that there is a $C_{p}^{\prime} \geq 0$ such that for all $f \in \mathscr{L}_{p}\left(\mathbb{B}_{n}\right),\|f\|_{\mathscr{R}^{1-p}} \leq C_{p}\|f\|_{\mathscr{R}^{1-p}}^{\Re} \leq$ $C_{p} C_{p}^{\prime}\|f\|_{\mathscr{L}_{p}}$, which is less than or equal to $C_{p} C_{p}^{\prime}\left(2+2 p^{-1}\right)\|f\|_{\mathscr{B}^{1-p}}$ by Lemma 3.3. The second statement in Theorem 3.5 follows.

\section{Proof of Theorem 2.1}

In the proof of Theorem 2.1, part (B), we will use part of the following lemma, which is obtained by straightforward estimates involving (1.8) (see [2, Chapter 4]). 
Lemma 4.1. Let $f \in \mathscr{H}(\mathbb{D})$, where $\mathbb{D}$ is an open subset of $\mathbb{C}^{n}$, and suppose that $\mathbb{U}$ is an orthonormal basis for $\mathbb{C}^{n}$. Then for all $z \in \mathbb{D}$,

$$
\left|\nabla^{\mathbb{U}} f(z)\right| \asymp|\nabla f(z)|
$$

We are now ready to prove Theorem 2.1.

Proof of Theorem 2.1. (A) Suppose that for some $M \geq 0$,

$$
\frac{\left(1-|z|^{2}\right)^{q}}{\left(1-|\phi(z)|^{2}\right)^{p}}\left|\nabla \phi_{j}(z)\right| \leq M \quad \forall z \in \mathbb{B}_{n}, j \in\{1,2, \ldots, n\}
$$

If $z \in \mathbb{B}_{n}$ and $F(z)=\left(1-|z|^{2}\right)^{q}\left|\nabla\left(\mathfrak{C}_{\phi} f\right)(z)\right|$, then we have that

$$
\begin{aligned}
F(z) & =\left(1-|z|^{2}\right)^{q} \sqrt{\sum_{i=1}^{n}\left|D_{i}(f \circ \phi)(z)\right|^{2}} \leq\left(1-|z|^{2}\right)^{q} \sum_{i=1}^{n}\left|D_{i}(f \circ \phi)(z)\right| \\
& \leq\left(1-|z|^{2}\right)^{q} n \sum_{j=1}^{n}|\nabla f(\phi(z))|\left|\nabla \phi_{j}(z)\right| \\
& =n|\nabla f(\phi(z))|\left(1-|\phi(z)|^{2}\right)^{p} \frac{\left(1-|z|^{2}\right)^{q}}{\left(1-|\phi(z)|^{2}\right)^{p}} \sum_{j=1}^{n}\left|\nabla \phi_{j}(z)\right| \\
& \leq n \sup _{w \in \mathbb{B}_{n}}\left\{|\nabla f(w)|\left(1-|w|^{2}\right)^{p}\right\} \sum_{j=1}^{n} \frac{\left(1-|z|^{2}\right)^{q}}{\left(1-|\phi(z)|^{2}\right)^{p}}\left|\nabla \phi_{j}(z)\right| \leq n\|f\|_{\oiint_{B} p} n M,
\end{aligned}
$$

by inequality (4.2). It follows that $\left\|\mathfrak{C}_{\phi} f\right\|_{\mathscr{R}^{q}} \leq\left(C+n^{2} M\right)\|f\|_{\mathscr{B}^{p}}$ for every $f \in \mathscr{S}^{P}\left(\mathbb{B}_{n}\right)$, thus completing the proof of Theorem 2.1, part (A).

(B) We proceed by modifying the argument given in [4, pages 187-188] for $n=1$. For $a \in \mathbb{B}_{n}$, define $f_{a}: \mathbb{B}_{n} \rightarrow \mathbb{C}$ to be the function that vanishes at 0 and is an antiderivative of $\psi_{a}: \mathbb{B}_{n} \rightarrow \mathbb{C}$ given by $\psi_{a}(t)=(1-\bar{a} t)^{-p}$. Let $w \in \mathbb{B}_{n}$ and $u \in \partial \mathbb{B}_{n}$. Define $F_{w, u}: \mathbb{B}_{n} \rightarrow \mathbb{C}$ by

$$
F_{w, u}(z)=f_{\langle w, u\rangle}(\langle z, u\rangle)
$$

Define $\phi_{u}: \mathbb{B}_{n} \rightarrow \mathbb{B}_{1}$ by $\phi_{u}(z)=\langle\phi(z), u\rangle$. Let $u^{(1)}:=u$, and choose $u^{(2)}, u^{(3)}, \ldots, u^{(n)}$ so that $\mathbb{U}=\left\{u^{(1)}, u^{(2)}, u^{(3)}, \ldots, u^{(n)}\right\}$ is an orthonormal basis for $\mathbb{C}^{n}$. For all $z \in \mathbb{B}_{n}$ and $j \in$ $\{2,3, \ldots, n\}$, we have that

$$
\begin{aligned}
D_{u^{(j)}} F_{w, u}(z) & =\lim _{\lambda \rightarrow 0} \frac{F_{w, u}\left(z+\lambda u^{(j)}\right)-F_{w, u}(z)}{\lambda} \\
& =\lim _{\lambda \rightarrow 0} \frac{f_{\left\langle w, u^{(1)}\right\rangle}\left(\left\langle z+\lambda u^{(j)}, u^{(1)}\right\rangle\right)-f_{\left\langle w, u^{(1)}\right\rangle}\left(\left\langle z, u^{(1)}\right\rangle\right)}{\lambda}=0 .
\end{aligned}
$$


On the other hand, for every $z \in \mathbb{B}_{n}$,

$$
\begin{aligned}
D_{u^{(1)}} F_{w, u}(z) & =\lim _{\lambda \rightarrow 0} \frac{F_{w, u}(z+\lambda u)-F_{w, u}(z)}{\lambda} \\
& =\lim _{\lambda \rightarrow 0} \frac{f_{\langle w, u\rangle}(\langle z, u\rangle+\lambda)-f_{\langle w, u\rangle}(\langle z, u\rangle)}{\lambda}=\psi_{\langle w, u\rangle}(\langle z, u\rangle) .
\end{aligned}
$$

From (4.5) and (4.6), it follows that

$$
\left|\nabla^{\mathbb{U}} F_{w, u}(z)\right|=|\psi\langle w, u\rangle(\langle z, u\rangle)|=|1-\overline{\langle w, u\rangle}\langle z, u\rangle|^{-p} .
$$

We observe that the quantity above is bounded when $u$ is fixed. This fact and Lemma 4.1 together imply that $F_{w, u} \in \mathscr{B}_{0}^{p}\left(\mathbb{B}_{n}\right)$. Also, we have

$$
F_{w, u}(0)=f_{\langle w, u\rangle}(\langle 0, u\rangle)=f_{\langle w, u\rangle}(0)=0 .
$$

Furthermore, by Lemma 4.1, we have that

$$
\begin{aligned}
\sup _{z \in \mathbb{B}_{n}}\left(1-|z|^{2}\right)^{p}\left|\nabla F_{w, u}(z)\right| & =\sup _{z \in \mathbb{B}_{n}}\left(1-|z|^{2}\right)^{p}\left|\nabla^{\mathbb{U}} F_{w, u}(z)\right| \\
& =\sup _{z \in \mathbb{B}_{n}}\left(1-|z|^{2}\right)^{p}|1-\overline{\langle w, u\rangle}\langle z, u\rangle|^{-p} .
\end{aligned}
$$

Note that

$$
|1-\overline{\langle w, u\rangle}\langle z, u\rangle|^{-p} \leq(1-|z|)^{-p} \leq \frac{2^{p}}{\left(1-|z|^{2}\right)^{p}} .
$$

It follows that the quantity (4.9) is less than or equal to $2^{p}$. Hence, $F_{w, u} \in \mathscr{B}^{P}\left(\mathbb{B}_{n}\right)$ for every $w \in \mathbb{B}_{n}$ and $u \in \partial \mathbb{B}_{n} ;$ moreover, the set

$$
\left\{\left\|F_{w, u}\right\|_{\Re^{p}}: u \in \partial \mathbb{B}_{n}, w \in \mathbb{B}_{n}\right\}
$$

is bounded. This fact and the hypothesis together imply that there exist $C$ and $M \geq 0$ such that for every $w \in \mathbb{B}_{n}$ and $u \in \partial \mathbb{B}_{n}$,

$$
\left\|F_{w, u} \circ \phi\right\|_{\Re^{q}} \leq C\left\|F_{w, u}\right\|_{\Re^{p}} \leq C M .
$$

Therefore, we obtain that

$$
\sup _{u \in \partial \mathbb{B}_{n}, z, w \in \mathbb{B}_{n}}\left\{\left|\nabla\left(f_{\langle w, u\rangle} \circ \phi_{u}\right)(z)\right|\left(1-|z|^{2}\right)^{q}\right\} \leq C M .
$$


Now for each $j \in\{1,2, \ldots, n\}$, we have that

$$
\begin{aligned}
D_{j}\left(f_{\langle w, u\rangle} \circ \phi_{u}\right)(z) & =f_{\langle w, u\rangle}^{\prime}(\langle\phi(z), u\rangle) D_{j}\langle\phi(z), u\rangle \\
& =(1-\overline{\langle w, u\rangle}\langle\phi(z), u\rangle)^{-p} D_{j}\langle\phi(z), u\rangle .
\end{aligned}
$$

It follows that

$$
\nabla\left(f_{\langle w, u\rangle} \circ \phi_{u}\right)(z)=(1-\overline{\langle w, u\rangle}\langle\phi(z), u\rangle)^{-p} \nabla\langle\phi(z), u\rangle .
$$

Using (4.15), we can rewrite (4.13) as

$$
\sup _{u \in \partial \mathbb{B}_{n}, z, w \in \mathbb{B}_{n}} \frac{\left(1-|z|^{2}\right)^{q}}{|1-\overline{\langle w, u\rangle}\langle\phi(z), u\rangle|^{p}}|\nabla\langle\phi(z), u\rangle| \leq C M .
$$

In particular, we have that

$$
\sup _{u \in \partial \mathbb{B}_{n}, z \in \mathbb{B}_{n}} \frac{\left(1-|z|^{2}\right)^{q}}{\left(1-|\langle\phi(z), u\rangle|^{2}\right)^{p}}|\nabla\langle\phi(z), u\rangle| \leq C M
$$

from which the statement of Theorem 2.1, part (B), follows.

By restricting the values of $u$, one obtains various necessary conditions for boundedness of $C_{\phi}$ from part (B) of Theorem 2.1. Two of such conditions are listed in Corollary 4.2 below. We point out that the boundedness of quantity (4.18) below when $\mathfrak{C}_{\phi}$ is bounded from $\mathscr{B}^{p}\left(\mathbb{B}_{n}\right)$ to $\mathscr{B}^{q}\left(\mathbb{B}_{n}\right)$ is a result given by Zhou in [11].

Corollary 4.2. Let $p, q>0$. If $\mathfrak{C}_{\phi}$ is a bounded operator from $\mathscr{B}^{p}\left(\mathbb{B}_{n}\right)$ (resp., $\mathscr{B}_{0}^{p}\left(\mathbb{B}_{n}\right)$ ) to $\mathscr{B}^{q}\left(\mathbb{B}_{n}\right)$, then there is an $M \geq 0$ such that the following statements hold.

(i) For all $z \in \mathbb{B}_{n}$ with $\phi(z) \neq 0$,

$$
\frac{\left(1-|z|^{2}\right)^{q}}{\left(1-|\phi(z)|^{2}\right)^{p}} \frac{\left|J_{\phi}(z)^{T} \cdot \phi(z)\right|}{|\phi(z)|} \leq M .
$$

(ii) For all $z \in \mathbb{B}_{n}$ and $j \in\{1,2, \ldots, n\}$,

$$
\frac{\left(1-|z|^{2}\right)^{q}}{\left(1-\left|\phi_{j}(z)\right|^{2}\right)^{p}}\left|\nabla \phi_{j}(z)\right| \leq M
$$

Proof. Putting $u:=\overline{\phi(z)} /|\phi(z)|$ in Theorem 2.1, part (B), one obtains that quantity (4.18) is no larger than some $M^{\prime} \geq 0$ for all $z \in B_{n}$ such that $\phi(z) \neq 0$. Successively replacing $u \in \partial \mathbb{B}_{n}$ in Theorem 2.1, part (B), by the typically ordered standard basis elements $e_{j}$ of $\mathbb{C}^{n}$ for $j=1,2, \ldots, n$, we see that the left side of inequality (4.19) is no larger than some $M^{\prime \prime} \geq 0$, so that we can choose $M:=\max \left(M^{\prime}, M^{\prime \prime}\right)$. 


\section{Acknowledgment}

The authors would like to thank W. Wogen for kindly pointing out that an earlier coordinate-dependent version of Theorem 2.1, part (B), could be improved to its current coordinate-free form.

\section{References}

[1] B. R. Choe, Projections, the weighted Bergman spaces, and the Bloch space, Proceedings of the American Mathematical Society 108 (1990), no. 1, 127-136.

[2] D. D. Clahane, Composition operators on holomorphic function spaces of several compex variables, Ph.D. thesis, University of California, Irvine, 2000.

[3] D. D. Clahane, S. Stević, and Z. Zhou, Composition operators on general Bloch spaces of the polydisk, preprint, 2004, http://arxiv.org/abs/math.CV/0506424.

[4] C. C. Cowen and B. D. MacCluer, Composition Operators on Spaces of Analytic Functions, Studies in Advanced Mathematics, CRC Press, Florida, 1995.

[5] P. L. Duren, Theory of $H^{p}$ Spaces, Pure and Applied Mathematics, vol. 38, Academic Press, New York, 1970.

[6] G. H. Hardy and J. E. Littlewood, Some properties of fractional integrals. II, Mathematische Zeitschrift 34 (1932), no. 1, 403-439.

[7] K. M. Madigan, Composition operators on analytic Lipschitz spaces, Proceedings of the American Mathematical Society 119 (1993), no. 2, 465-473.

[8] W. Rudin, Function Theory in the Unit Ball of $\mathbb{C}^{n}$, Fundamental Principles of Mathematical Science, vol. 241, Springer, New York, 1980.

[9] S. Stević, On an integral operator on the unit ball in $\mathbf{C}^{n}$, Journal of Inequalities and Applications 2005 (2005), no. 1, 81-88.

[10] W. Yang and C. Ouyang, Exact location of $\alpha$-Bloch spaces in $L_{a}^{p}$ and $H^{p}$ of a complex unit ball, The Rocky Mountain Journal of Mathematics 30 (2000), no. 3, 1151-1169.

[11] Z. Zhou and H. Zeng, Composition operators between p-Bloch and q-Bloch space in the unit ball, Progress in Natural Science. English Edition 13 (2003), no. 3, 233-236.

[12] K. Zhu, Spaces of Holomorphic Functions in the Unit Ball, Graduate Texts in Mathematics, vol. 226, Springer, New York, 2005.

Dana D. Clahane: Department of Mathematics, University of California, Riverside, CA 92521, USA

E-mail address: dclahane@math.ucr.edu

Stevo Stević: Mathematical Institute of the Serbian Academy of Science, Knez Mihailova 35/I,

11000 Beograd, Serbia

E-mail addresses: sstevic@ptt.yu; sstevo@matf.bg.ac.yu 